Cahiers
Recherche
surles Droits Fondamentaux
Cahiers de la recherche sur les droits fondamentaux

17 | 2019

La motivation des actes administratifs. Le droit français à la lumière du droit administratif comparé

\title{
La motivation des actes administratifs en Italie
}

\section{Antonio Cassatella}

\section{(2) OpenEdition}

Journals

Édition électronique

URL : https://journals.openedition.org/crdf/3745

DOI : $10.4000 /$ crdf.3745

ISSN : 2264-1246

Éditeur

Presses universitaires de Caen

Édition imprimée

Date de publication : 31 décembre 2019

Pagination : 99-108

ISBN : 978-2-84133-960-0

ISSN : $1634-8842$

Référence électronique

Antonio Cassatella, "La motivation des actes administratifs en Italie », Cahiers de la recherche sur les droits fondamentaux [En ligne], 17 | 2019, mis en ligne le 06 février 2021, consulté le 14 novembre 2022. URL : http://journals.openedition.org/crdf/3745 ; DOI : https://doi.org/10.4000/crdf.3745 


\title{
La motivation des actes administratifs en Italie
}

\author{
Antonio CASSATELLA \\ Professeur associé de droit administratif à la faculté de droit de l'université de Trente (Italie)
}

\begin{abstract}
I. L'évolution théorique et pratique de la motivation aux $\mathrm{XIX}^{\mathrm{e}}$ et $\mathrm{XX} \mathrm{X}^{\mathrm{e}}$ siècles
II. La structure et les fonctions de la motivation dans la loi $n^{0} 241 / 1990$

III. Les vices de la motivation et le contrôle du juge

IV. Les problèmes actuels

V. Conclusions
\end{abstract}

\section{L'évolution théorique et pratique de la motivation aux $\mathrm{XIX}^{\mathrm{e}}$ et $\mathrm{XX} \mathrm{X}^{\mathrm{e}}$ siècles}

La motivation des actes administratifs représente un problème pratique et théorique étudié aussi bien du point de vue de la jurisprudence que de la doctrine ${ }^{1}$.

$\mathrm{Au}$ cours du XIX ${ }^{\mathrm{e}}$ siècle et presque tout au long du XX, ce concept n'a fait l'objet d'aucune loi générale, laissant ainsi à la jurisprudence et à la doctrine la tâche de combler les lacunes de la législation quant à la détermination de la nécessité de la motivation. Il n'existait d'ailleurs que quelques lois spéciales imposant la motivation de certains actes. Ces lois n'exprimaient toutefois pas un principe général valable pour les cas non prévus par les textes. L'exemple le plus important est celui de l'article 3 de la loi no 2248/1865, annexe E, qui obligeait l'administration à motiver ses décisions lors des recours préalables: même si une partie de la doctrine a tenté de lui attribuer une portée générale en proposant une interprétation par analogie, la jurisprudence n'a jamais adopté cette thèse ${ }^{2}$. L'absence d'une loi générale n'a permis ni à la jurisprudence ni à la doctrine d'atteindre un consensus sur la structure et les fonctions de la motivation. Ces sujets ont fait l'objet de longs débats qui ne semblent pas avoir abouti malgré la codification du concept par l'article 3 de la loi nº 241/1990.

Les études les plus récentes ont démontré comment quatre modèles théoriques de la motivation ont pu s'imposer à partir de la jurisprudence et des tentatives de rationalisation de la doctrine ${ }^{3}$. Au cours des XIX et $\mathrm{XX}^{\mathrm{e}}$ siècles, ces modèles théoriques se sont superposés suivant l'évolution qui a affecté le droit administratif italien ainsi que ses concepts les plus importants: l'acte administratif, la procédure administrative, la justice administrative. À leur tour, ces modèles théoriques sont le résultat des transformations de l'idée d'administration et de son rôle à l'intérieur d'un État de droit constitutionnel, non seulement dans ses rapports avec ses citoyens mais aussi avec l'ordre économique et social en général ${ }^{4}$.

1. Pour une toute première introduction, voir. A. Cassatella, Il dovere di motivazione nell'attività amministrativa, Padoue, CEDAM, 2013; A. Cassatella, "Motivazione del provvedimento amministrativo", in Diritto on line, Rome, Istituto dell'Enciclopedia Italiana Treccani, 2018, en ligne: http:// www.treccani.it/enciclopedia/motivazione-del-provvedimento-amministrativo_\%28Diritto-on-line\%29; F. Aperio Bella, «La motivazione del provvedimento », in Principi e regole dell'azione amministrativa, M. A. Sandulli (dir.), Milan, Giuffrè, 2015, p. 203 sq.; F. Cardarelli, «La motivazione del provvedimento", in Codice dell'azione amministrativa, M. A. Sandulli (dir.), $2^{\mathrm{e}}$ éd., Milan, Giuffrè, 2017, p. 377 sq. ; A. Cioffi, « La motivazione del provvedimento amministrativo», in L'azione amministrativa, A. Romano (dir.), Turin, G. Giappichelli, 2016, p. 199 sq.; B. Marchetti, «Il principio di motivazione", in Studi sui principi del diritto amministrativo, M. Renna, F. Saitta (dir.), Milan, Giuffrè, 2012, p. 521 sq.

2. Voir F. Benvenuti, «Funzione amministrativa, procedimento, processo», Rivista trimestrale di diritto pubblico, 1952, p. 118 sq.

3. Voir A. Cassatella, Il dovere di motivazione nell'attività amministrativa, p. 97 sq.

4. Voir M. S. Giannini, Il pubblico potere. Stati e amministrazioni pubbliche, Bologne, Il mulino, 1986. 
Le premier modèle théorique est de nature volontaristica ou «volontariste» et il s'est diffusé dans les premières décennies du XIX ${ }^{\mathrm{e}}$ siècle. L'idée initiale de ce modèle était que l'acte administratif était l'expression de la «volonté» de l'administration. Toute l'activité administrative était donc conçue à l'image de la volonté de la personne physique ayant qualité d'autorité administrative au sein de l'organisme. Cette analogie amenait à conclure à la nonpertinence de l'expression des motifs de l'acte administratif dans la mesure où les motifs étaient considérés comme non pertinents pour exprimer la volonté contractuelle elle-même ${ }^{5}$. Ainsi, la jurisprudence ne considérait pas la motivation comme nécessaire dans les cas où la loi ne l'exigeait pas $^{6}$. Cette conception ressemblait au principe «pas de motivation sans texte» qui s'imposa dans le droit français ${ }^{7}$ pendant la même période.

Toutefois, dans un second temps, la doctrine et la jurisprudence ont considéré que, pour les actes administratifs (unilatéraux), il fallait appliquer un principe contraire à celui du droit privé: alors que l'expression des motifs était inutile quant à la détermination de la volonté contractuelle, ils étaient par contre toujours essentiels quant à la détermination de l'acte administratif (unilatéral) ${ }^{8}$. La solution proposée n'avait aucun fondement théorique réel mais elle s'expliquait par la nécessité de protéger le citoyen contre tout acte défavorable. Il s'agissait plutôt d'une exigence politique: si l'acte administratif n'est pas motivé, on s'expose au risque d'abus de pouvoir ou de renforcement de l'autoritarisme dans le sens où la volonté vaut raison (stat pro ratione voluntas). Au début des années 1940, cette thèse fut développée dans le cadre du modèle théorique relazionale ou «relationnel». La motivation de l'acte défavorable était considérée comme une garantie essentielle en faveur de tous les sujets qui avaient une relation avec l'administration et notamment des destinataires d'actes défavorables ${ }^{9}$.

Contrairement au modèle volontariste, cette thèse ne faisait pas appel à la théorie de l'acte administratif comme acte d'expression de la volonté de l'administration. Elle justifiait la nécessité de la motivation en se référant à d'autres qualités de l'acte administratif telle que sa valeur contraignante et unilatérale ${ }^{10}$. La motivation compensait donc les privilèges dont jouissait l'administration dans l'accomplissement de ses missions car elle imposait le respect de la garantie procédurale par l'obligation d'indiquer clairement le processus logique et l'argumentation à la base de tout acte administratif. Selon cette idée, la motivation pouvait être vue comme un corollaire du principe du clare loqui qui fonde l'idée moderne de transparence administrative ${ }^{11}$. La notion la plus claire de la motivation a été fournie par Guido Zanobini pour lequel c'est « la prise en compte des considérations d'ordre juridique, technique et administratif qui justifient l'adoption de telle mesure $»^{12}$.

Il en résultait que, même en l'absence d'une loi sur la procédure administrative, l'administration était obligée de motiver tout acte défavorable au citoyen. À la lumière de la motivation écrite, le citoyen aurait pu librement vérifier l'opportunité d'une saisine juridictionnelle en vue d'obtenir l'annulation de l'acte. Ce modèle de nature protectrice était également fort utile au niveau pratique: en imposant à l'administration de motiver tout acte défavorable, on accordait au juge administratif un pouvoir de contrôle juridictionnel sur la légalité de l'acte fondée sur le texte même de la mesure. Cette solution était judicieuse car elle limitait fortement les moyens d'instruction du juge qui ne pouvait ni connaître ni évaluer directement les faits pris en compte dans la procédure administrative.

Cette thèse a été par la suite pleinement consacrée par l'article 24 de la Constitution italienne de 1948 : d'un point de vue fonctionnel, l'obligation de la motivation renvoyait à l'exercice du droit fondamental de se défendre reconnu au citoyen face à l'administration. La limite du modèle relationnel tenait à ce qu'il se concentrait exclusivement sur la défense du citoyen face à l'administration. Il ne se préoccupait nullement de l'utilité de la motivation en dehors des hypothèses où l'action administrative n'est pas contestée.

À partir des années 1960, l'importance de la motivation s'est renforcée dans le domaine de la procédure administrative, considérée comme une façon d'appliquer la loi sur la base de la théorie Stufenbau élaborée par Kelsen et Merkl dans les années $1920^{13}$. Selon ce modèle dinamico

5. Voir O. Ranelletti, «Capacità e volontà nelle autorizzazioni e concessioni amministrative» [1894], in Scritti giuridici scelti, E. Ferrari, B. Sordi (éd.), Naples, Jovene, 1992, p. 177 sq. ; F. Cammeo, "Gli atti amministrativi e l'obbligo di motivazione», Giurisprudenza Italiana, III, 1908, p. 253 sq.

6. Sur la jurisprudence, voir G. Bergonzini, La motivazione degli atti amministrativi, Vicence, s.n., 1979.

7. Pour une analyse comparée voir A. Cassatella, Il dovere di motivazione nell'attività amministrativa, p. 163 sq.; A. Cassatella, «Il dovere di motivazione nello jus commune europeo", in I procedimenti amministrativi di adjudication nell'Unione europea: principi generali e discipline settoriali, G. Della Cananea, M. Conticelli (dir.), Turin, G. Giappichelli, 2017, p. 17 sq.

8. Voir CE italien, IV, 17 mai 1907, nº 178, où le devoir de motivation a été identifié par rapport à la «nature» de l'acte. Dans la doctrine, ce modèle a été développé surtout par C. M. Iaccarino, Studi sulla motivazione: con speciale riguardo agli atti amministrativi, Rome, Il Foro italiano, 1933; A. M. Sandulli, Il procedimento amministrativo, Milan, Giuffrè, 1940.

9. Sur les fondements philosophiques de cette idée, voir G. Del Vecchio, Lezioni di filosofia del diritto, 13 ${ }^{\mathrm{e}}$ éd., Milan, Giuffrè, 1957; A. Levi, Istituzioni di teoria generale del diritto, Padoue, CEDAM, 1934.

10. Voir G. Miele, «L’obbligo di motivazione degli atti amministrativi » [1941], in Scritti giuridici, Milan, Giuffrè, 1987, p. 329 sq. ; C. Mortati, « Obbligo di motivazione e sufficienza della motivazione degli atti amministrativi», Giurisprudenza Italiana, III, 1943, p. 7 sq. ; G. Roherssen, « Note sulla motivazione degli atti amministrativi", Rivista di diritto pubblico, 1941, p. 124 sq.

11. Sur le critère du clare loqui, voir aussi CE italien, V, 14 avril 2008, $\mathrm{n}^{\circ} 1665$; CE italien, $\mathrm{V}, 5$ juillet $2007, \mathrm{n}^{\circ} 3819$; CE italien, IV, 14 février $2005, \mathrm{n}^{\circ} 435$

12. Voir G. Zanobini, Corso di diritto amministrativo. I. Principi generali, $8^{\mathrm{e}}$ éd., Milan, Giuffrè, 1958, p. 279 sq.

13. Voir U. Allegretti, L'imparzialità amministrativa, Padoue, CEDAM, 1965; G. Berti, «La struttura procedimentale dell'amministrazione pubblica», Diritto e società, 1980, p. 437 sq. ; La procedura amministrativa, G. Pastori (dir.), Venise, N. Pozza, 1964; G. Pastori, «Il procedimento amministrativo tra vincoli formali e regole sostanziali ", in Diritto amministrativo e giustizia amministrativa nel bilancio di un decennio di giurisprudenza, 
ou "dynamique», la motivation n'est pas seulement importante afin de garantir le contrôle juridictionnel de l'acte administratif, mais également pour protéger la rationalité et la transparence de la procédure administrative à son tour considérée comme concrétisation de la loi. La motivation n'est pas utile pour le seul destinataire de l'acte défavorable ou pour le juge, mais pour l'administration aussi qui sera obligée d'agir de façon rationnelle et impartiale déjà au cours de la procédure. En ce sens, la motivation peut garantir la légalité substantielle de l'acte administratif. La motivation n'est pas simplement un élément de l'acte administratif, mais c'est une étape de toute l'activité de l'administration. Elle permet par conséquent de mettre en application les valeurs exprimées dans l'article 97, alinéa 2, de la Constitution italienne en garantissant l'impartialité et la bonne administration. Cette théorie n'a pas d'effets pratiques immédiats mais elle représente la base théorique de l'article 3 de la loi $n^{\circ} 241 / 1990$ (loi générale sur la procédure administrative) qui exige la motivation de tous les actes administratifs en tant que garantie des principes d'impartialité, de publicité et de transparence soulignés par l'article 1 de la loi, en application de l'article 97 de la Constitution.

Si ces différents modèles ne s'opposent pas entre eux mais représentent plutôt le développement progressif d'une conception de nature protectrice de la motivation, une autre thèse critiquant l'utilité même de ce concept s'est imposée dans les années 1970 en Italie. Il s'agit ici d'un modèle pluralistico ou "pluraliste» qui distingue l'importance juridique de la motivation formelle de celle de la motivation substantielle ${ }^{14}$. On pensait alors que la loi et la jurisprudence avaient identifié de manière très claire la plupart des cas où la motivation formelle, c'est-à-dire l'expression écrite des motifs de l'acte, était nécessaire: ainsi en était-il pour le retrait et l'abrogation de l'acte administratif, les sanctions administratives, les ordres de police, les décrets d'expropriation, les refus opposés aux demandes des particuliers, ou encore tout acte discrétionnaire ${ }^{15}$.

Les débats sur ce point étaient donc vains et de moindre importance. Il fallait concentrer l'analyse sur d'autres problèmes. Dans l'activité de l'administration, le moment de la «décision» était plus important que celui de la motiva- tion $^{16}$ : dans la décision on effectuait un choix visant l'intérêt public qui allait ultérieurement être motivé. La motivation formelle était habituellement une rationalisation a posteriori d'un choix accompli lors de la procédure. C'était parfois un artifice qui empêchait de connaître les vrais motifs de l'acte administratif et qui provoquait la confusion aussi bien dans l'esprit des citoyens que dans celui du juge. Même l'annulation de l'acte administratif pour vice de motivation était peu utile pour la partie requérante car dans la plupart des cas l'administration conservait le pouvoir de promulguer un nouvel acte au contenu identique ou comparable à celui qui avait été annulé mais en le motivant de façon plus claire.

En Italie, on critique cette conception en ayant recours à l'image assez connue de la victoire à la Pyrrhus ${ }^{17}$. Selon cette doctrine, par conséquent, le citoyen et le juge devaient pouvoir connaître la motivation substantielle de l'acte, c'est-à-dire l'ensemble des faits identifiés et des évaluations effectuées lors de la phase d'instruction de la procédure administrative. Le problème de la codification générale de la motivation de tous les actes défavorables était ici de moindre importance et l'on se concentrait plutôt sur le rôle que ce concept jouait dans le domaine du contrôle du juge ${ }^{18}$. On critiquait les juges qui se limitaient à contrôler la logique et la cohérence du texte de la motivation de chaque acte.

Cette thèse, marquée par un réalisme évident, a été critiquée au cours des années $1980^{19}$. Si la motivation formelle n'était pas, en termes absolus, une garantie de la rationalité, de la transparence et de l'impartialité de l'activité administrative, ceci ne signifiait pas qu'il fallait la restreindre au profit de la motivation substantielle. L'idée même de motivation substantielle était vague: le formalisme qui caractérise l'activité administrative et qui oblige l'administration à documenter toutes les étapes de la procédure implique que la motivation substantielle s'exprime dans un acte écrit. Il n'existe donc aucune motivation qui puisse renoncer à toute explication pertinente. Il en découle que la motivation formelle ne peut avoir une vraie fonction de garantie que si elle porte sur la motivation substantielle. Le contraire n'est pas vrai: aucune motivation substantielle, en l'absence d'une motivation formelle, n'est suffisante pour comprendre la décision

U. Allegretti, A. Orsi Battaglini, D. Sorace (dir.), Rimini, Maggioli Editore, 1987, t. II, p. 805 sq. Pour une analyse des fondements théoriques de cette idée, qui s'appuie sur les études de Feliciano Benvenuti (1916-1999) sur la procédure administrative, voir M. Bellavista, "Giusto processo come garanzia del giusto procedimento", in Procedura, procedimento, processo, L. R. Perfetti (dir.), Milan, CEDAM, 2010 , p. 249 sq.

14. Voir M. S. Giannini, «Motivazione dell'atto amministrativo», in Enciclopedia del diritto, Milan, Giuffrè, 1977, t. XXVII, p. 265 sq. ; R. Iannotta, "La motivazione come modo di attuazione del principio di imparzialità amministrativa ", in Aspetti e tendenze del diritto costituzionale. Scritti in onore di Costantino Mortati, Milan, Giuffrè, 1977, p. 199 sq.

15. Voir R. Juso, Motivi e motivazione nel provvedimento amministrativo, Milan, Giuffrè, 1963, p. 79 sq.

16. Sur la décision comme possibilité de choix entre plusieurs alternatives, voir M. Nigro, Studi sulla funzione organizzatrice della pubblica amministrazione, Milan, Giuffrè, 1966, p. 87 sq. Sur cette doctrine, voir aussi M. Bombardelli, Decisioni e pubblica amministrazione. La determinazione procedimentale dell'interesse pubblico, Turin, G. Giappichelli, 1996; A. Police, La predeterminazione delle decisioni amministrative. Gradualità e trasparenza nell'esercizio del potere discrezionale, Naples, Edizioni scientifiche italiane, 1997.

17. Voir M. Nigro, "Sulla riproduzione dell'atto amministrativo annullato per difetto di motivazione» [1958], in Scritti giuridici, Milan, Giuffrè, 1996, t. I, p. 441 sq. ; A. Piras, «Intervento», in Aspetti e problemi dell'esercizio del potere di sostituzione nei confronti dell'amministrazione locale, Milan, Giuffrè, 1983 , p. 56 sq.

18. Voir M. S. Giannini, Il potere discrezionale della pubblica amministrazione. Concetto e problemi, Milan, Giuffrè, 1939.

19. Voir A. Romano Tassone, Motivazione dei provvedimenti amministrativi e sindacato di legittimità, Milan, Giuffrè, 1987 , p. 191 sq.; G. Corso, «Motivazione dell'atto amministrativo», in Enciclopedia del diritto. Aggiornamento, Milan, Giuffrè, 2001, t. V, p. 775 sq. 
de l'administration ${ }^{20}$. On risque, en effet, sur la base de certains éléments de fait et de droit qui constituent la motivation substantielle, d'établir des interprétations différentes qui ne sont pas toujours transparentes ou rationnelles. Cette observation ne doit pas surprendre. La critique la plus efficace avait déjà été exprimée dans le contexte de la théorie générale du droit, par Emilio Betti: faisant référence à la motivation de tous les actes juridiques, Betti avait bien expliqué sans équivoque comment

[...] l'exactitude, la justesse de la motivation est aussi importante qu'une décision légitime et juste: une mesure qui décide bien mais réfléchit mal ne satisfait ni les objectifs que la loi lui a attribués ni le «sentiment de justice» ${ }^{21}$.

Même la doctrine la plus récente a affirmé à quel point la motivation est un instrument essentiel pour garantir la rationalité de l'action administrative considérée en elle-même ainsi que la protection des droits individuels face à l'administration. On a ajouté que la motivation écrite de tout acte administratif est fondamentale afin que l'administration assume ses responsabilités lorsqu'elle produit certains effets juridiques et parvient à des résultats pratiques $^{22}$.

La Cour constitutionnelle est arrivée à la même conclusion lorsqu'elle a défini en 2015 la motivation comme «la condition préalable, le fondement, le centre de gravité et l'essence même de l'exercice légitime du pouvoir administratif et, pour cela, la base substantielle et incontournable de la légalité ${ }^{23}$.

\section{La structure et les fonctions de la motivation dans la loi $n^{0} 241 / 1990$}

La conception relationnelle et la conception dynamique ont inspiré l'action du législateur qui a codifié le devoir de motivation des actes administratifs en 1990 dans l'article 3 de la loi $n^{\circ}$ 241/1990. La disposition figure au chapitre I de la loi $n^{\circ}$ 241/1990 consacré aux principes de l'action administrative. Le devoir de motiver représente donc un principe général de l'action administrative de toutes les institutions publiques, qui réalise aussi bien les valeurs définies dans les articles 24 et 97 de la Constitution que les autres principes clairement définis dans l'article 1 de la loi $n^{\circ} 241 / 1990$, tels que ceux de publicité et de transparence.
L'insertion de l'article 3 de la loi no 241/199o parmi les normes de principe de l'action administrative reflète la jurisprudence constitutionnelle qui considère la motivation comme facteur de modération des risques d'arbitraire administratif et comme corollaire des principes généraux du système juridique ${ }^{24}$. Pour mieux comprendre l'importance actuelle de la motivation dans l'ordre juridique italien il faut en analyser les caractéristiques structurelles et fonctionnelles ${ }^{25}$.

En ce qui concerne les caractéristiques structurelles, la motivation est une étape de la procédure mais aussi un élément de l'acte. L'article 3, alinéa 1, de la loi nº 241/1990 établit que «tout provvedimento doit être motivé». Par "provvedimento", l'on entend l'acte unilatéral par lequel se termine la procédure administrative et qui a des effets juridiques sur le destinataire et les tiers. Lors de la formation du provvedimento, l'administration doit motiver ses choix selon des actions bien précises ${ }^{26}$. Les caractéristiques de ces actions sont précisées, toujours à l'article 3, alinéa 1 , de la loi no 241/1990 : la motivation consiste à identifier les motifs de fait et de droit qui ont conduit à la décision administrative suite à l'instruction effectuée au cours de la procédure ${ }^{27}$. On a vu comment l'article 3 de la loi $n^{\circ} 241 / 1990$ impose à l'administration de motiver son propre choix à la fin de l'instruction et de la formation de la décision à assumer. Ceci implique que cette action précède la formulation définitive de l'acte unilatéral en tant qu'acte final produisant des effets juridiques ${ }^{28}$.

Le devoir de motivation consiste donc à passer en revue la phase d'instruction et les modalités suivies par l'administration pour parvenir à une décision, afin de sélectionner les motifs de fait et de droit ayant eu une répercussion immédiate et directe sur les solutions possibles. Le respect du devoir de motivation implique l'élaboration d'un document faisant partie de la structure même de l'acte administratif qui clôt la procédure.

Du point de vue structurel, la motivation peut être considérée comme la partie de l'acte qui précède le dispositif et qui indique quels éléments de l'instruction ont déterminé, aussi bien en fait qu'en droit, un certain choix et la décision qui en résulte. L'article 3 de la loi nº 241/1990 reconnaît par conséquent l'importance de la motivation formelle conçue comme partie du provvedimento et précise qu'elle doit consister en une motivation substantielle de la décision (c'est-à-dire les motifs préalables de fait et les

20. Voir F. Ledda, «Problema amministrativo e partecipazione al procedimento» [1993], in Scritti giuridici, Padoue, CEDAM, 2002, p. 348 sq. ; M. Nigro, «Procedimento amministrativo e tutela giurisdizionale contro la pubblica amministrazione (il problema di una legge generale sul procedimento amministrativo)» [1980], in Scritti giuridici, Milan, Giuffrè, 1996, t. II, p. 1442 sq.

21. Voir E. Betti, Interpretazione della legge e degli atti giuridici, Milan, Giuffrè, 1949, p. 237. Toutes les traductions de l'italien sont de nous.

22. Voir A. Cassatella, Il dovere di motivazione nell'attività amministrativa, p. 275 sq.

23. Voir Cour constitutionnelle italienne, ordonnance, 29 avril 2015, $\mathrm{n}^{\circ} 92$.

24. Voir Cour constitutionnelle italienne, 2 novembre $2010, \mathrm{n}^{\circ} 310$.

25. Voir M. Ramajoli, «L'esigenza sistematica nel diritto amministrativo attuale», Rivista trimestrale di diritto pubblico, 2010, p. 347 sq.

26. Sur la distinction entre acte et mesure, voir R. Villata, M. Ramajoli, Il provvedimento amministrativo, $2^{\mathrm{e}}$ éd., Turin, G. Giappichelli, 2017 , p. 43 sq. L'acte unilatéral se distingue des mesures préparatoires, selon la loi n 241/199o. De plus, tous les actes unilatéraux doivent être motivés sans aucune distinction entre actes créateurs et actes non créateurs de droits.

27. Voir M. De Donno, «Riflessioni sulla "motivazione in diritto" del provvedimento amministrativo", Rivista trimestrale di diritto pubblico, 2013, p. 629 sq.

28. Voir A. Cassatella, Il dovere di motivazione nell'attività amministrativa, p. 280 sq. 
raisons juridiques). La jurisprudence elle-même souligne à cet égard que la motivation écrite doit toujours précéder le dispositif de l'acte. La motivation ne peut donc pas être élaborée après le dispositif, car dans ce cas-là elle risquerait de donner lieu à une justification a posteriori de la décision présumée reposer sur des raisons non avouées ${ }^{29}$.

La motivation ne peut être séparée de l'acte unilatéral que dans certains cas spécifiques: l'article 3, alinéa 3, de la loi $\mathrm{n}^{\circ}$ 241/199o permet, en effet, d'utiliser la motivazione per relationem (motivation par référence). Dans ce cas, l'acte peut être motivé en renvoyant à un autre acte motivé déjà émis au cours de la procédure par la même administration: cet autre acte doit alors être joint au nouvel acte. Par exemple, un ordre de démolition peut être motivé en renvoyant au contenu du procès-verbal dans lequel on a constaté la violation des normes de construction. Selon l'article 3 de la loi no 241/1990, la motivation est nécessaire pour tous les actes unilatéraux. La loi de 1990 a levé les incertitudes jurisprudentielles et doctrinales relatives à la motivation des actes relevant d'une compétence liée ${ }^{30}$. Il est désormais clair que la différence entre acte découlant de l'exercice d'une compétence discrétionnaire et acte découlant de l'exercice d'une compétence liée, ou encore entre acte favorable et défavorable, affecte seulement la portée de la motivation et les modes d'accomplissement de l'obligation imposée par l'article 3 de la loi n² 241/1990.

En revanche, la loi no 241/1990 n'explique pas comment motiver l'acte unilatéral et elle n'indique pas les conditions minimales d'une motivation «suffisante $»^{31}$. S'il est manifestement impossible de donner une suite favorable à la demande d'un particulier, l'article 2, alinéa 1, de la loi no 241/1990 permet de motiver l'acte sous une forme simplifiée par une référence synthétique aux circonstances de fait et de droit qui ont empêché de donner suite à la demande. Dans tous les autres cas, le niveau de suffisance de la motivation doit être déterminé en fonction des données acquises au cours de l'instruction. La portée de la motivation dépend, par conséquent, de la complexité de l'instruction et de la décision de l'administration.

Si l'administration doit adopter un acte unilatéral uniquement sur la base de lois tout à fait claires et précises, sans aucun pouvoir discrétionnaire, la motivation peut se limiter à indiquer les données de fait qui ont imposé l'application de la loi. Par exemple, il sera suffisant d'expliquer la raison pour laquelle un immeuble viole le règlement d'urbanisme pour en ordonner la démolition sur la base des prescriptions légales obligeant l'administration à sanctionner ladite violation. Si l'administration doit accomplir une série d'évaluations techniques afin de prendre une décision, la motivation devra expliquer la démarche suivie par les fonctionnaires pour vérifier et évaluer les faits. Il sera donc nécessaire d'assumer la responsabilité du choix du critère technique suivi pour en tirer une certaine conclusion. Par exemple, si la loi conditionne l'octroi d'une allocation dans le cas d'une maladie contractée par le salarié public dans le cadre de son propre travail, il sera nécessaire d'indiquer les preuves qui permettent d'établir un lien entre cette maladie et l'activité exercée par le salarié.

Si l'administration exerce un pouvoir discrétionnaire, la motivation devra indiquer les critères sur la base desquels on a privilégié un certain intérêt plutôt que d'autres. Étant donné que les décisions discrétionnaires de l'administration se fondent sur des évaluations d'adéquation, il sera nécessaire d'expliquer la raison pour laquelle telle décision a été considérée comme plus appropriée que d'autres solutions ${ }^{32}$.

Les aspects fonctionnels de la motivation ont évidemment plusieurs objectifs ${ }^{33}$. Vue sous l'angle de l'administration, la motivation fonctionne comme facteur de responsabilisation, ce qui nous ramène à la question de la responsabilité fonctionnelle des organes administratifs sur la base de l'article 97, alinéa 3, de la Constitution ${ }^{34}$. Toujours du point de vue de l'administration, elle fonctionne aussi comme un facteur de rationalisation de l'activité accomplie au cours de la procédure qui garantit le caractère raisonnable, l'impartialité et la proportionnalité des décisions ainsi que, par conséquent, la légalité substantielle de l'acte unilatéral sur la base de l'article 97, alinéa 2, de la Constitution. Vue sous l'angle du destinataire de l'acte, la motivation a pour but de rendre compréhensible la décision de l'administration dans l'hypothèse où une contestation de l'acte serait portée devant le juge, sur la base de l'article 24 de la Constitution. Vue sous l'angle du juge, la motivation représente les arguments textuels de la rationalité de la décision. Elle peut donc être utilisée pendant le procès comme indice de la légalité de l'acte contre lequel on a formé un pourvoi, même si ceci n'empêche pas le juge d'effectuer d'autres contrôles sur la base de tous les documents réunis lors du procès. Cette fonction aussi se fonde sur l'article 24 de la Constitution. Vue sous l'angle des organes politiques (par exemple, les ministres concernés), la motivation permet le contrôle et l'évaluation de l'activité des organes administratifs par rapport aux objectifs établis par les lois et les actes de programmation. Il s'agit d'une fonction justifiée par les articles 54, 95 et 98 de la Constitution ${ }^{35}$. Vue sous l'angle des citoyens qui ne sont pas les destinataires directs des actes, la motivation a pour but la transparence des décisions, dans la perspective d'un contrôle efficace de l'activité des pouvoirs publics.

29. Voir Conseil d'État italien, VI, 8 septembre 2017, nº 4253; Conseil d'État italien, III, 2 mai 2016, $\mathrm{n}^{\circ} 1656$.

30. Voir C. M. Iaccarino, Studi sulla motivazione..., p. 167 sq.

31. Voir C. Mortati, «Obbligo di motivazione...», p. 7 sq. ; A. Romano Tassone, Motivazione dei provvedimenti..., p. 366 sq. ; A. Cassatella, Il dovere di motivazione nell'attività amministrativa, p. 275 sq.

32. Voir R. Villata, M. Ramajoli, Il provvedimento amministrativo, p. 95 sq.

33. Voir A. Cassatella, Il dovere di motivazione nell'attività amministrativa, p. 264 sq.

34. Voir ibid.; A. Cassatella, «La responsabilità funzionale nell'amministrare. Termini e questioni », Diritto amministrativo, 2018, p. 677 sq.

35. Ces articles réglementent l'activité et la responsabilité du gouvernement et des fonctionnaires administratifs. 
Cette fonction est particulièrement importante par rapport aux décisions qui peuvent influencer le bien-être collectif, comme les actes promulgués dans le domaine de l'environnement ou de l'administration du territoire. Elle exprime l'exigence de contrôle démocratique de l'activité administrative, selon les articles 1, 2, 21 et 54 de la Constitution ${ }^{36}$.

Toutes les fonctions de la motivation identifiables sur la base de la Constitution peuvent renvoyer à l'exigence générale de bonne administration prévue par l'article 41 de la Charte des droits fondamentaux de l'union européenne ainsi qu'au principe du procès équitable prévu par l'article 6 de la Convention européenne des droits de l'homme ${ }^{37}$.

\section{Les vices de la motivation et le contrôle du juge}

La valeur juridique du devoir de motivation est étroitement liée à son incidence sur la validité de l'acte administratif et aux limites du contrôle judiciaire portant sur le contenu de la motivation. Si la violation du devoir de motiver prévue par l'article 3 de la loi n $241 / 1990$ n'avait aucune incidence effective sur la légalité de l'acte, l'article 3 serait tout à fait dépourvu de sens. Si on réfléchit de façon abstraite, il ne fait nul doute que la violation du devoir de motiver imposé par la loi devrait avoir comme conséquence l'invalidité de l'acte. Cependant, on pourrait affirmer que le vice de la motivation n'est pas un vice de la décision administrative: annuler un acte uniquement parce qu'il est motivé de façon imprécise est une conséquence excessive pour l'administration surtout si la décision est juste et appropriée ${ }^{38}$.

Le débat sur ce sujet est particulièrement vif en Italie depuis les années 1970, avec l'élaboration du modèle pluraliste. Si les thèses qui ont valorisé la fonction de garantie de la motivation imposent de considérer toujours l'acte insuffisamment motivé comme illégal, d'autres thèses ont critiqué la rigidité excessive de ces conclusions ${ }^{39}$. Contre la thèse mettant l'accent sur la fonction de garantie, s'est donc affirmée une thèse riduzionistica (restrictive). Selon cette dernière, si l'annulation de l'acte non motivé peut se révéler inutile pour la partie requérante, elle nuit aussi à l'efficacité de l'administration et de la justice administrative $^{40}$. Si l'on conteste en justice l'acte uniquement parce qu'il n'est pas motivé, le juge pourra en effet l'annuler, mais l'administration sera libre d'exercer son propre pouvoir en confirmant la décision prise en la motivant de manière plus approfondie. La partie requérante n'en tirera aucun bénéfice réel ${ }^{41}$. Dans cette hypothèse, on porte également préjudice à l'efficacité de l'administration. L'acte adopté, quoique dépourvu d'une motivation suffisante, peut être légitime du point de vue du contenu. La motivation peut être incomplète à cause de la superficialité et de la négligence du fonctionnaire, mais ceci n'affecte pas la légalité substantielle de la décision qui peut être concrètement la plus appropriée pour la protection de l'intérêt public. De plus, si l'acte est soumis à des dispositions légales précises, l'administration peut ne pas avoir de choix: l'acte n'est pas motivé mais la décision est tout à fait conforme à la loi en ce qui concerne son contenu ${ }^{42}$.

Un recours contre un acte caractérisé par une motivation insuffisante mais fondé sur une décision appropriée peut également affecter la gestion de la justice administrative car elle implique le déroulement de procès «inutiles». On a souvent affirmé ces dernières années à quel point le procès administratif est un instrument insuffisant et comme il est difficile d'imaginer que la protection juridictionnelle puisse être garantie pour satisfaire toute revendication de la partie requérante ${ }^{43}$ : la tenue d'un procès qui a pour seul objet la motivation formelle, dans les cas où la décision est fondamentalement appropriée, est non seulement inutile mais aussi excessivement coûteuse par rapport aux bénéfices sociaux qui pourraient en dériver. Le problème ne dépend donc pas de la nécessité de faire prévaloir la motivation substantielle sur la motivation formelle: on observe, toutefois, comment le contrôle de la motivation formelle est une condition nécessaire, mais non suffisante, pour évaluer la légalité de l'acte.

Les deux conceptions que nous venons de décrire peuvent être également justifiées sur la base de la loi. Les différentes conclusions de ces thèses dépendent par conséquent de la façon dont on interprète la loi, et des valeurs qui en orientent l'interprétation. Si l'on valorise

36. Il s'agit des articles concernant la protection des droits fondamentaux de l'individu dans l'État démocratique, la liberté d'expression de la pensée, le droit de participation à l'activité politique et administrative.

37. Voir A. Cassatella, Il dovere di motivazione nell'attività amministrativa, p. $206 \mathrm{sq}$

38. Voir M. S. Giannini, «Motivazione dell'atto amministrativo», p. 265 sq., selon lequel ce qui compte « ce n'est pas la motivation, mais uniquement le motif ou la condition préalable dans la réalité effective [...] ce qui compte, c'est ce qui a été fait, pas ce qu'on a déclaré vouloir faire».

39. Pour ces critiques, voir surtout M. S. Giannini, «Motivazione dell'atto amministrativo», p. 265 sq. ; R. Iannotta, «La motivazione come modo di attuazione...", p. 231 sq.

40. Voir toujours M. S. Giannini, «Motivazione dell'atto amministrativo», p. 265 sq. ; R. Iannotta, «La motivazione come modo di attuazione...», p. 231 sq. Il s'agit des mêmes chercheurs qui ont élaboré l'idée pluralistica de la motivation. Dans les textes les plus récents, c'est l'adhésion à une idée de garantie du droit administratif qui domine et le modèle pluraliste est vivement critiqué: voir A. Cassatella, Il dovere di motivazione nell'attività amministrativa, p. 27 sq. ; G. Mannucci, «Uno, nessuno, centomila. Le motivazioni del provvedimento amministrativo», Diritto pubblico, 2012, p. 837 sq.; M. Ramajoli, «Il declino della decisione motivata», Diritto processuale amministrativo, 2017, p. 894 sq.; G. Tropea, "Motivazione del provvedimento e giudizio sul rapporto", Persona e amministrazione, 2017, p. 239 sq.

41. Il s'agit du cas déjà indiqué de "victoire à la Pyrrhus».

42. Voir M. S. Giannini, «Motivazione dell'atto amministrativo», p. 265 sq.

43. Voir La giustizia amministrativa come servizio (tra effettività ed efficienza), G. Comporti (dir.), Florence, Firenze University Press, 2016; S. Licciardello, «La giustizia amministrativa come servizio pubblico», Diritto processuale amministrativo, 2015, p. 797 sq. ; A. Pajno, «Giustizia amministrativa ed economia», Diritto processuale amministrativo, 2015, p. 952 sq. 
la dimension de garantie du droit administratif, on considérera toujours comme illégal et annulable tout acte non motivé. Si l'on valorise par contre la dimension d'efficacité, l'acte non motivé sera annulable uniquement s'il peut en dériver des bénéfices pour la partie requérante. L'article 21, octies, alinéa 1, de la loi nº 241/199o explique comment les actes administratifs sont annulables en cas de violation de la loi: la violation du devoir de motivation équivaut à la violation de l'article 3 de la loi no $241 / 1990$ et devrait toujours avoir comme résultat l'annulation de l'acte. Selon la jurisprudence et la doctrine traditionnelles, l'annulation est à prévoir aussi bien dans les rares situations où la motivation formelle est tout à fait absente que dans les cas où elle existe, mais est exprimée de manière insuffisante $^{44}$. En cas de motivation insuffisante, on affirme que la décision administrative est arbitraire: en ce qui concerne les actes discrétionnaires, une jurisprudence soutient depuis toujours qu'il s'agit d'un indice de détournement de pouvoir ${ }^{45}$. Cette manière de raisonner a toutefois été démentie par une partie de la jurisprudence selon laquelle le juge ne doit pas se limiter à contrôler si la motivation formelle de l'acte est insuffisante, mais doit aussi évaluer si la décision est logique par rapport au déroulement de toute la procédure administrative. Il s'agit de thèses qui proposent les mêmes idées que celles qui fondent l'idée pluraliste élaborée par la doctrine dans les années 1970 mais mise à jour au vu des exigences sociales actuelles. Le Conseil d'État a soutenu, par exemple, que le devoir de motivation est satisfait lorsque

[...] les règles de la procédure sont soigneusement suivies de sorte qu'on puisse raisonnablement affirmer que les organes publics ont agi sans anticiper le résultat final de leur action, excluant ainsi partialité et inefficacité ${ }^{46}$.

Mais cette conclusion fait référence à la motivation substantielle de l'acte, c'est-à-dire à la pertinence des raisons juridiques qui sont à la base du choix accompli par l'administration: si la motivation substantielle est appropriée, les vices de la motivation formelle peuvent affecter uniquement la façon dont on explique la décision, mais pas la décision elle-même. Concrètement, ceci implique que la partie requérante ne devrait pas affirmer l'illégalité de l'acte seulement parce que celui-ci manque d'une motivation formelle insuffisante. Elle doit également contester d'autres vices de l'instruction et de la décision. Le juge ne devrait pas se contenter de vérifier les vices du texte de la motivation de l'acte, mais il devrait également élargir sa connaissance au contenu de la décision. L'annulation de l'acte ne devrait pas être prévue uniquement en cas d'insuffisance de la motivation formelle, mais aussi en cas d'autres vices affectant la motivation et la décision (par exemple, quand la motivation formelle concerne des situations de fait inexistantes ou des décisions contraires au principe de proportionnalité). À partir de ces idées, une partie de la jurisprudence a affirmé, ces dernières années, que la violation de l'article 3 de la loi no 241/1990 n'est qu'un vice de procédure qui implique l'irrégularité de l'acte, sur la base de l'article 21, octies, alinéa 2, de la loi no $241 / 1990^{47}$.

Cette thèse ne se limite pas à affirmer qu'en cas de vice de la motivation formelle l'acte n'est pas annulable, mais elle reconnaît de même que l'administration peut donner, au cours du procès, toutes les précisions et explications utiles à justifier sa propre décision ${ }^{48}$. Il s'agit, cependant, d'une thèse qui a été fortement critiquée non seulement par la doctrine, mais aussi par la jurisprudence administrative dominante et par la Cour Constitutionnelle: on estime en effet que l'article 21, octies, alinéa 2, de la loi $n^{\circ} 241 / 1990$ est inapplicable en cas de vices de la motivation et qu'il doit être réservé aux autres vices de procédure ${ }^{49}$.

Si on veut essayer de simplifier la description de la situation italienne, on peut indiquer les aspects essentiels des effets des vices de la motivation sur la légalité du provvedimento par rapport aux fonctions de la motivation qu'on vient de décrire. C'est la partie requérante qui choisit de contester uniquement le vice de motivation formelle. Elle est donc libre de contester la validité de l'acte pour tout vice de légalité sur la base des principes établis par les articles 24 et 113 de la Constitution italienne. Dans ce cas, la partie requérante doit être consciente des risques d'aboutir à une «victoire à la Pyrrhus ", mais son autonomie ne peut nullement être conditionnée par des raisonnements liés à l'efficacité de l'administration et de la justice administrative: ces considérations sont sans aucun doute importantes, mais au niveau constitutionnel elles n'ont pas la même valeur que le droit au contrôle juridictionnel de l'administration qui constitue un droit fondamental du citoyen. Toutefois, la partie requérante ne se limite pas à contester l'acte uniquement parce qu'il est non motivé. Dans la pratique judiciaire, en effet, l'acte est contesté pour plusieurs vices de légalité qui concernent en général toute la phase d'instruction et de décision de l'administration: quand la partie requérante affirme que l'acte est caractérisé par une motivation insuffisante,

44. Selon la doctrine et la jurisprudence, l'absence de motivation ne détermine pas la nullité de l'acte sur le fondement de l'article 21, septies, de la loi n ${ }^{\circ}$ 241/199o. Contre cette idée, voir au contraire A. Cassatella, Il dovere di motivazione nell'attività amministrativa, p. 284 sq., où l'on affirme que, lorsque la motivation est absente, c'est un élément essentiel de l'acte qui manque et l'acte est donc nul.

45. Voir Conseil d'État italien, IV, 7 janvier $1892, \mathrm{n}^{\circ} 3$.

46. Voir Conseil d'État italien, IV, 27 janvier 2012, $n^{\circ} 429$.

47. Selon l'article 21, octies, alinéa 2, l'acte lié n'est pas annulable pour vices de forme ou de procédure si l'administration démontre, en justice, qu'on ne pouvait pas décider autrement. Sur les vices de procédure, voir D. U. Galetta, Violazione di norme sul procedimento amministrativo e annullabilità del provvedimento, Milan, Giuffrè, 2003; Vizi formali, procedimento e processo amministrativo, V. Parisio (dir.), Milan, Giuffrè, 2004, p. 71 sq.

48. Voir Conseil d'État italien, IV, 4 mars 2014, nº 1018; Conseil d'État italien, IV, 9 octobre 2012, n ${ }^{\circ} 5257$.

49. Voir A. Cassatella, Il dovere di motivazione nell'attività amministrativa, p. 273 sq. ; G. Mannucci, «Uno, nessuno, centomila...", p. 837 sq.; M. Ramajoli, «Il declino...», p. 894 sq. 
ceci signifie que la décision aussi est fausse, car les vices de motivation font ressortir toute erreur de fait et de droit $^{50}$. Il est, en revanche, interdit à l'administration de se défendre en justice avec des explications visant à justifier a posteriori un acte caractérisé par une motivation sans doute insuffisante: concrètement, ce problème pourrait affecter les actes discrétionnaires qu'il faut absolument annuler si l'administration n'a pas donné, lors de l'adoption de l'acte, une explication appropriée et complète des faits et des raisons juridiques à la base de sa décision.

\section{Les problèmes actuels}

Les questions concernant l'importance procédurale n'épuisent pas les problèmes concernant la motivation. D'autres problèmes plus actuels concernent en effet ces aspects qui ne sont pas pris en compte par la loi $n^{\circ} 241 / 1990$ mais qui surgissent quand il s'agit de l'activité administrative dans le droit italien.

Le premier problème concerne le domaine d'application de la motivation : l'article 3, alinéa 2, de la loi $n^{\circ} 241 / 1990$ exclut en effet les actes réglementaires et les lois du devoir de motivation. Si le problème de la motivation des lois concerne le droit constitutionnel ${ }^{51}$, celui des actes réglementaires concerne quelques actes administratifs particulièrement importants: l'article 3 , alinéa 2, exclut en effet du champ de la motivation des actes tels que les plans, les programmes, les règlements ${ }^{52}$. L'explication de cette exclusion a toujours été fondée sur les effets des actes généraux: ces actes n'ont pas d'influence directe sur la position des citoyens car seuls les actes individuels pris pour l'exécution des plans et des programmes sont susceptibles d'affecter les intérêts de ces derniers. Par conséquent, dans une perspective de garantie, il était suffisant d'imposer la motivation des seuls actes individuels et non celle des plans ni des programmes afin de permettre aux citoyens de contester en justice les actes unilatéraux adoptés.

Cette solution n'est toutefois pas satisfaisante, si l'on reconnaît que la motivation peut être utile pour l'administration même, et pour les citoyens désireux de connaître et d'évaluer les choix sous-jacents à tout acte administratif, mais aussi pour les dirigeants politico-administratifs. On a donc accepté, dans la doctrine et dans la jurisprudence, que l'article 3, alinéa 2, de la loi nº 241/199o ne devait pas interdire de réglementer la motivation des actes administratifs à travers des lois spéciales spécifiques ${ }^{53}$. De tels actes ont souvent un contenu discrétionnaire, ils peuvent affecter la jouissance de plusieurs droits et la réalisation de plusieurs intérêts publics et ils doivent donc être élaborés de sorte à éviter que leur contenu soit arbitraire ou leurs choix peu transparents. Cette exigence est en conformité avec les principes du droit européen dont l'article $296 \mathrm{du}$ traité sur le fonctionnement de l'Union européenne pose l'obligation de motiver les directives et les règlements. Ceci explique, d'ailleurs, pourquoi dans les lois spéciales de ces vingt dernières années le devoir de motiver s'est progressivement répandu, surtout pour ce qui concerne en particulier les actes des autorités indépendantes et certains plans d'aménagement du territoire ${ }^{54}$. Toutefois, l'absence d'une disposition législative générale en la matière s'avère assez grave. En effet, l'article 3, alinéa 2, de la loi $n^{\circ}$ 241/1990 ne satisfait pas les exigences actuelles tendant à l'extension de l'obligation de motivation aux actes réglementaires et constitue, au contraire, une limite à la définition de règles destinées à établir le contenu ou le caractère suffisant de la motivation de tels actes. Il est certain, toutefois, que l'article 3 , alinéa 2, ne permet pas d'élargir aux actes généraux les dispositions de l'alinéa 1, lesquelles sont explicitement applicables aux seuls actes qui ont un destinataire spécifique.

Une autre question controversée dans la pratique est celle de la possibilité de motiver certains actes en "forma numerica ", c'est-à-dire en recourant à des chiffres plutôt que sous la forme rédigée en toutes lettres ${ }^{55}$. Par exemple, l'évaluation d'une épreuve écrite pour obtenir l'admission à l'exercice de la profession d'avocat est motivée tout simplement avec des chiffres qui expriment le niveau de suffisance ou d'insuffisance atteint; un autre exemple peut être celui de la formation d'un classement suite à une procédure d'adjudication pour un contrat public ${ }^{56}$. L'article 3, alinéa 1, de la loi no 241/1990 n'exige pas de manière explicite que la motivation soit écrite en italien et laisse au contraire la possibilité de motiver certains actes sur la base de chiffres. La jurisprudence administrative et la Cour constitutionnelle ont estimé que la motivation sous forme de chiffres pouvait être légale tout au moins lorsque

50. Voir A. Cassatella, Il dovere di motivazione nell'attività amministrativa, p. 43 sq.

51. Voir S. Boccalatte, La motivazione della legge. Profili teorici e giurisprudenziali, Padoue, CEDAM, 20o8; M. Picchi, L'obbligo di motivazione delle leggi, Milan, Giuffrè, 2011.

52. Voir T. Bonetti, «La deroga all'obbligo di motivare gli atti a contenuto generale: vent'anni dopo », in La legge sul procedimento amministrativo: vent'anni dopo, A. Sandulli, G. Piperata (dir.), Naples, Editoriale scientifica, 2011, p. 339 sq. ; M. Cocconi, «L'obbligo di motivazione degli atti amministrativi generali», Rivista trimestrale di diritto pubblico, 2009, p. 730 sq. ; M. C. Romano, "L'esclusione della motivazione per gli atti normativi e per gli atti a contenuto generale", in L'azione amministrativa..., p. 231 sq.

53. Voir A. Cassatella, Il dovere di motivazione nell'attività amministrativa, p. 364 sq.

54. Voir A. Cassatella, «La motivazione dei piani urbanistici: aspetti evolutivi», Rivista giuridica dell'edilizia, 2016, p. 289 sq.; V. Mazzarelli, «La motivazione delle varianti urbanistiche», Giornale di diritto amministrativo, 2007, p. 1174 sq. ; R. Montefusco, «La motivazione degli atti di pianificazione urbanistica : atti amministrativi generali ? ", Foro amministrativo, 2002, p. 2861 sq.

55. Voir A. Cassatella, Il dovere di motivazione nell'attività amministrativa, p. 340 sq. ; M. L. Maddalena, "Voto numerico e obbligo di motivazione», Corriere del merito, 2011, p. 897 sq.

56. Voir A. Cassatella, «La valutazione dell'offerta economicamente più vantaggiosa e il dovere di motivazione : note critiche », in Gli appalti pubblici tra regole europee e nazionali, G. Benacchio, M. Cozzio (dir.), Milan, EGEA, 2012, p. 375 sq. 
les actes dépendent de l'application de critères de jugement rigides et donc en l'absence de tout pouvoir discrétionnaire de l'administration ${ }^{57}$. La doctrine, par contre, est beaucoup plus critique envers cette solution, car elle juge que seule une motivation discursive et écrite en langue italienne est vraiment transparente et en mesure d'expliquer au destinataire de l'acte les raisons d'un jugement négatif ${ }^{58}$. La raison pratique pour laquelle la jurisprudence italienne continue d'admettre la motivation en chiffres ne dépend pas, toutefois, du fait que la motivation soit réellement en mesure d'expliquer clairement le sens d'un certain acte: quoique peu utile pour le destinataire, ce genre de motivation est très utile pour conclure dans un délai raisonnable les concours et les appels d'offres publics.

Même par rapport à ce problème, l'article 3 de la loi $n^{\circ}$ 241/1990 n'est pas formulé de façon acceptable. La meilleure solution serait de motiver les actes administratifs qui expriment un jugement de suffisance ou d'insuffisance d'une épreuve en appliquant des algorithmes mathématiques. Par exemple, la motivation ne serait pas nécessaire pour expliquer l'échec d'un candidat dont les réponses à un test à choix multiples étaient fausses. Dans tous les autres cas, au contraire, la motivation écrite en langue italienne serait nécessaire: par exemple, l'insuffisance à une épreuve de droit administratif devrait être expliquée en relevant les erreurs du candidat et leur gravité, etc.

Un autre aspect problématique concerne le rapport entre le règlement général et le règlement spécial de l'activité administrative, là où on donne la possibilité aux lois spéciales ou sectorielles (et aux lois régionales) de déroger à l'article 3 de la loi n $241 / 1990$, en interdisant ou en limitant la motivation de chaque acte unilatéral.

Il apparaît donc, d'après la jurisprudence de la Cour constitutionnelle, que les dérogations peuvent concerner la forme et le mode de formulation de la motivation, mais elles ne doivent jamais interdire la motivation de chaque acte, même si ladite interdiction pourrait être fondée sur la bonne administration. Toutefois la jurisprudence n'empêche pas que chaque loi puisse prévoir des régimes de motivation spécifiques selon les caractéristiques des actes et des destinataires ${ }^{59}$

\section{Conclusions}

Dans de précédentes études de droit comparé consacrées à la motivation des actes administratifs, on a pu mettre en évidence les caractéristiques de la situation italienne par rapport à celle des autres législations ${ }^{60}$. Il s'agit d'un système où la codification du devoir de motivation dans la loi no 241/1990 a rendu la jurisprudence et la doctrine plus respectueuses des garanties du citoyens face à l'emploi arbitraire de tout pouvoir, indépendamment de la nature discrétionnaire de l'acte.

L'article 3 de la loi $n^{\circ}$ 241/199o est conforme aux valeurs constitutionnelles mais aussi aux principes de droit européen élaborés par la Cour de justice et réaffirmés dans l'article 41 de la charte de Nice, ainsi que dans l'article 6 de la Convention européenne des droits de l'homme et dans la jurisprudence de la Cour européenne des droits de l'homme. La jurisprudence de la Cour constitutionnelle italienne a souvent fait remarquer l'importance du devoir de motivation et ceci explique pourquoi, dans l'ordre juridique italien, il n'y a jamais eu de résistance à reconnaître la dimension de garantie de la motivation prévue aussi dans le droit européen.

Il s'agit d'une des rares situations où le droit administratif italien semble avoir assumé - du moins dans la perspective des chercheurs de droit interne - un rôle de premier plan aussi bien dans l'élaboration théorique de la motivation que dans l'analyse étendue et continue de son importance. Ceci, non seulement pour ce qui concerne la théorie de l'acte administratif, de la procédure et du contrôle juridictionnel mais aussi par rapport à la légitimité démocratique de l'activité administrative et aux toutes récentes questions concernant la responsabilité fonctionnelle des organes administratifs.

Ces données sont sans aucun doute positives, mais il ne faut pas oublier qu'une partie de la jurisprudence ainsi qu'une partie de la doctrine mettent en évidence les limites de cette insistance sur la fonction de garantie de la motivation: les juges et les chercheurs les plus réalistes affirment que la légitimité de l'action administrative doit être trouvée dans la procédure et dans la décision finale et pas dans le provvedimento ou dans sa motivation formelle. Rechercher dans la motivation une garantie contre l'arbitraire administratif, un instrument de légitimité démocratique et de transparence administrative, peut être très dangereux dans la pratique et dans la culture juridique italienne. Les fonctionnaires de l'administration italienne ayant reçu une formation juridique sont en effet très adroits à motiver de façon convaincante une décision, mais cela ne signifie pas que ce qui est écrit soit vrai ou vraisemblable. La motivation peut donc devenir une sorte de «voile» qui cache les vérités apparues lors de la procédure. Pour l'ensemble de ces raisons, on insiste sur la nécessité de ne pas limiter le contrôle juridictionnel ainsi que les évaluations de l'opinion publique à ce qui est écrit dans la motivation mais plutôt de concentrer l'analyse sur les contenus de la décision. Ceci implique, dans une

57. Voir Cour constitutionnelle italienne, 8 juin 2011, $\mathrm{n}^{\circ}$ 375, selon laquelle «le critère (en chiffres) répond aux exigences du bon fonctionnement de l'action administrative, qui n'impliquent pas, de la part des commissions d'examen, une explication détaillée des raisons ayant conduit à un jugement de non-admissibilité, eu égard aux délais des opérations du concours ou d'habilitation et au nombre de candidats aux épreuves». Conseil d'État italien, IV, 31 août 2018, nº 5129; Conseil d'État italien, VI, 7 mai 2018, nº 2699

58. Voir A. Cassatella, Il dovere di motivazione nell'attività amministrativa, p. 340 sq.

59. Voir Cour constitutionnelle italienne, 2 novembre 2010, $\mathrm{n}^{\circ} 310$.

60. Voir A. Cassatella, Il dovere di motivazione nell'attività amministrativa, p. 163 sq. ; A. Cassatella, «Il dovere di motivazione nello jus commune europeo». 
perspective critique et réaliste, qu'il faut faire attention à la substance de l'activité plutôt qu'à sa forme juridique.

Il faut, toutefois, émettre des doutes sur l'analyse des sceptiques. Certes, la motivation n'est certainement pas une garantie absolue de rationalité, elle n'est pas forcément véridique ou en mesure de représenter le contenu d'une décision. Néanmoins, l'existence d'un devoir général de motiver l'acte impose aux juristes de prendre au sérieux les dispositions de l'article 3 de la loi no 241/1990 et les valeurs qu'elles expriment, ceci valant aussi pour la Cour constitutionnelle. Les administrations doivent prendre très au sérieux ce devoir, parce qu'il décourage toute décision illégale ou déraisonnable, et encourage par contre à assumer une responsabilité manifeste en ce qui concerne les effets de l'acte qui est l'expression du choix le plus approprié.
Les critiques concernant le devoir de motivation et la façon dont il est parfois employé par les administrations peuvent être significatives au niveau de la sociologie et de la science administrative ou des théories qui refusent la valeur normative du droit et de la science juridique. Toutefois, si l'on admet que la législation, la jurisprudence et la science juridique ont un sens et leur propre autonomie, dans le sens qu'elles expriment une idée normative du droit qui met en évidence «le devoir être» inscrit dans le phénomène juridique, on doit conclure que l'accomplissement du devoir de motiver continue d'être une condition nécessaire et indispensable de l'action administrative (stat pro voluntate ratio).

À défaut, on risque le retour d'une idée autoritaire du pouvoir (stat pro ratione voluntas). 